\title{
Identification, cloning and characterization of an ultrapetala transcription factor CsULT1 from Crocus: a novel regulator of apocarotenoid biosynthesis
}

\author{
Nasheeman Ashraf ${ }^{* *}$, Deepti Jain² and Ram A Vishwakarma ${ }^{3}$
}

\begin{abstract}
Background: Crocus sativus is a triploid sterile plant with long red stigmas which form commercial saffron. Saffron is the site for synthesis and accumulation of apocarotenoids like crocin, picrocrin and safranal which are responsible for its color, flavour and aroma making it world's most expensive spice. These compounds are formed by oxidative cleavage of zeaxanthin by carotenoid cleavage dioxygenases. Although the biosynthetic pathway of apocarotenoids is known to a considerable extent, the mechanism that regulates its tissue and developmental stage specific expression is not known.

Results: In the present work, we identified, cloned and characterized ultrapetala transcription factor called CsULT1 from Crocus. The gene contains an 80 amino acid long conserved SAND domain. The CsULT1 transcript was more abundant in stigma and showed increase in expression from pre anthesis stage till anthesis and decreased in post anthesis stage which corroborated with the accumulation pattern of crocin indicating its possible role in regulation of apocarotenoid biosynthesis. CSULT1 was found to be transcriptionally active and localized in nucleus. Its expression is induced in response to phytohormones like auxin, methyljasmonate and salicylic acid. Overexpression of CSULT1 in Crocus calli resulted in enhanced expression of key pathway genes like phytoene synthase (PSY), phytoene desaturase (PDS), beta carotene hydroxylase $(B C H)$ and carotenoid cleavage dioxygenases (CCDS) indicating its role in regulation of apocarotenoid biosynthesis.
\end{abstract}

Conclusion: This work presents first report on isolation and characterization of ultrapetala gene from Crocus. Our results suggest that CSULT1 is a novel regulator of Crocus apocarotenoid biosynthesis. We show for the first time involvement of plant SAND domain proteins in regulating secondary metabolic pathways.

Keywords: Ultrapetala, Crocus, Stigma, Saffron, Carotenoids, Apocarotenoids, SAND domain

\section{Background}

Crocus sativus L. (Iridaceae) is a sterile triploid plant propagated vegetatively through corms [1]. The desiccated stigma of $C$. sativus forms saffron and is source of various carotenoids and unique compounds called apocarotenoids which are produced by oxidative tailoring of carotenoids [2]. Apocarotenoids are synthesized in a number of plants including maize, tomato, Arabidopsis,

\footnotetext{
*Correspondence: nashraf@iiim.ac.in

'Plant Biotechnology Division, CSIR-Indian Institute of Integrative Medicine, Sanat Nagar, Srinagar J\&K-190005, India

Full list of author information is available at the end of the article
}

Crocus etc. but Crocus finds a special place because it is the only plant which produces crocin, picrocrocin and saffranal in significant quantities [3]. The saffron apocarotenoids are formed by zeaxanthin cleavage [4] followed by specific glycosylation steps [5]. Because of the presence of these unique apocarotenoids Crocus stands apart from other related crops and is considered as one of the world's costliest spices [6]. Besides, saffron apocarotenoids also have tremendous pharmacological properties and have been used for the treatment of a wide range of cancers $[7,8]$. 
Carotenoids and their cleavage products are synthesized by plastid localized methylerythritol phosphate (MEP) pathway. Biosynthesis of these compounds is regulated throughout the life cycle of a plant and dynamic changes occur in their composition to match the prevailing developmental requirements and response to external environmental stimuli [9]. Although the carotenoid biosynthetic pathway has been studied to a considerable extent in many plants including Crocus but the fundamental knowledge regarding the regulation of carotenogenesis in plant cells is still in its infancy [10]. In Crocus, apocarotenoids are synthesized only in stigma part of the flower and that too in developmental stage specific manner, but nothing is known about the mechanism that regulates its synthesis. Therefore, it will be quite interesting to take a step towards unravelling the regulatory pathway of carotenoid/apocarotenoid biosynthesis in Crocus.

The major goal of the present study was to identify transcription factors that regulate apocarotenoid biosynthesis in saffron. It is a well established fact that stigma part of the Crocus flower is the actual site for synthesis of many important apocarotenoids [11,12], however, we still attempted to study pattern of crocin accumulation (crocin being an important metabolite) in different parts of the flower and at different stages of stigma development. We also selected five transcription factors belonging to five different gene families from saffron gene database [1] and investigated their temporal and spatial expression profile. The results demonstrated that ULTRAPETALA (ULT) gene shows higher expression in stigma tissue and the expression increased till the day of anthesis and subsequently decreased. This expression profile matched with the accumulation pattern of crocin in saffron thereby indicating a possible role of this gene in regulating biosynthesis of apocarotenoids. The ULT encodes a small cysteïne rich protein containing a B-box like motif and a SAND domain, a DNA binding motif previously reported only in animal transcription factors [13]. This transcription factor has been proposed to act as regulator of developmental gene expression. In Arabidopsis, it functions in floral stem cell termination pathway [14]. ULT has been shown to act as trithorax group chromatin remodelling factor which regulates function of Agamous locus during stem cell termination [15]. Recently it has been demonstrated that ULT1 acts as an antirepressor that promotes transcriptional activation by antagonizing PcG-induced histone methylation and, via physical interaction with ATX1 that deposits H3K4me3 activating marks, promotes an open chromatin conformation to recruit proteins involved in transcriptional initiation and elongation [16]. More recently $U L T$ was found to be involved in gynoecium formation [17]. ULT1 function thus represents a novel chromatin-mediated mechanism that activates genes controlling stem cell fate in plants. This observation expands the repertoire of plant epigenetic regulators involved in developmental pathways and suggests involvement of chromatin mediated pathways in controlling dynamics of transcription during such pathways.

In this report we describe identification, isolation and characterization of ULT gene, CsULT1, from Crocus sativus. CsULT1 is preferentially expressed in stigma and induced by phytohormones such as MJ, SA, 2,4-D. Further, CsULT1 is localized in nucleus and is transcriptionally active. Crocus transformation has not yet been established. Here we studied transient overexpression of CsULT1 in Crocus calli and observed that its overexpression upregulates some key carotenoid/apocarotenoid pathway genes. This work represents, to our knowledge, the first functional characterization of a C. sativus ULT gene and also first report on a transcriptional regulator of apocarotenoid biosynthetic pathway.

\section{Methods}

\section{Plant material}

Crocus sativus was grown in the experimental farm at Indian Institute of Integrative medicine (IIIM), Srinagar, India (longitude: $34^{\circ} 5^{\prime} 24^{\prime \prime} \mathrm{N}$; latitude: $74^{\circ} 47^{\prime} 24^{\prime \prime}$ and altitude $1585 \mathrm{~m}$ above sea level). It was used as source plant material for the present study. The voucher specimen was deposited at Janaki Ammal Herbarium (RRLH), IIIM, Jammu. The details of the specimen are: (Accession number: 22893; Accession date: 12/01/2015; name of collector: Nasheeman Ashraf; Place of collection: IIIM, Srinagar Farm; Date of collection: 01/01/2015). For tissue specific expression profiling, on the day of flower opening, tepals, anthers and stigma were collected from flowers separately, frozen in liquid nitrogen and stored in $-80^{\circ} \mathrm{C}$ till further use. For developmental stage specific expression, stigma was collected at three different stages viz three days before anthesis, on the day of anthesis and two day after anthesis. For hormone treatments, flowers were grown in pots and were mist sprayed with $100 \mu \mathrm{M}$ methyljasmonate, $1 \mathrm{mM}$ salicylic acid, $50 \mu \mathrm{M}$ 2, 4-D and $100 \mu \mathrm{M}$ ABA. Tissue samples were collected after 12 and $24 \mathrm{~h}$ of hormone treatment. For overexpression studies, calli overexpressing CsULT1 and vector control calli were taken for RNA isolation. For each experiment, tissue from three biological replicates was pooled in.

\section{Sample preparation and HPLC analysis}

Crocin analysis was done as described by Moraga et al. [12]. For extract preparation, $0.5 \mathrm{mg}$ tissue from tepals, anthers and stigma (collected at three different stages) was crushed with a micropestle in $700 \mu \mathrm{l}$ Tris- $\mathrm{HCl}$ (50 mM, pH 7.5 containing $1 \mathrm{M} \mathrm{NaCl}$ ), and incubated for 10 minutes on ice. This was followed by addition of 
$700 \mu \mathrm{l}$ of chloroform. The extract was then incubated on ice for an additional $10 \mathrm{~min}$. Centrifugation at $3000 \mathrm{~g}$ for $5 \mathrm{~min}$ at $4^{\circ} \mathrm{C}$ was done to separate the phases. The lower chloroform phase was evaporated and the dried residues were stored together with the upper aqueous phases at $-80^{\circ} \mathrm{C}$ until high-performance liquid chromatography (HPLC) analysis. The LCMS apparatus of Nexera UHPLC (130 MPa) equipped with MS-8030 (Shimadzu) was used for the Study and data was generated using lab solutions software. Enable RP-C18 column $(250 \mathrm{~mm} \times 4.6 \mathrm{~mm}, 5 \mu \mathrm{m})$ was used. The injection volume was $5 \mu \mathrm{l}$ and flow rate $0.3 \mathrm{ml} / \mathrm{min}$. Mobile Phase A (Water and Acetonitrile ratio 1:1) and mobile phase B $(0.1 \%$ Acetic acid in water) were used in a linear gradient flow and column temperature was set at $75^{\circ} \mathrm{C}$ initially.

Gene expression analysis using quantitative real time PCR Total RNA was extracted from pooled tissue using TRIzol reagent and used for cDNA synthesis by Reverse Transcription kit (Fermentas) following manufacturer's instructions. qRT-PCR was performed in triplicates in ABI StepOne Real time (Applied biosystems). The reaction was carried out in a total volume of $20 \mu \mathrm{l}$, consisting of $10 \mu \mathrm{L}$ of $2 \mathrm{X}$ SYBR Green Master Mix, $0.2 \mu \mathrm{M}$ (each) gene specific primers for all the genes studied and $100 \mathrm{ng}$ of template cDNA. The cycling parameters were $95^{\circ} \mathrm{C}$ for $20 \mathrm{~s}$, followed by 40 cycles of $95^{\circ} \mathrm{C}$ for $15 \mathrm{~s}$ and $60^{\circ} \mathrm{C}$ for $1 \mathrm{~min}$. The sequence of all the primers used in this study is given in Additional file 1. The specificity of each primer pair was validated by a dissociation curve (a single peak was observed for each primer pair) (Additional file 2). The relative quantification method $(\Delta \Delta-C T)$ was used to evaluate quantitative variation between the replicates examined. The amplification of actin cDNA was used as an endogenous control to normalize all data.

\section{Cloning of full length CsULT1 gene}

The partial clone of CsULT1 was obtained using cDNA synthesized from Crocus flower RNA and primers (ULT-F and ULT-R) designed from EST sequence (cr.saCl000502:1) present in NCBI (www.ncbi.nlm.nih.gov/nucest). Sequence analysis of the partial clone revealed that it has the 3 'end and only 5'end needs to be amplified in order to obtain the full length clone. Thus the full length cDNA clone of CSULT1 was obtained by performing 5'RACE using gene specific primer (ULT-5') and UAP primer provided with the 5'RACE kit (Clontech) following manufacturer's instructions. The amplified product was run on $1 \%$ agarose gel and purified with gel extraction kit (Qiagen). The purified product was then cloned in the pGEM-T Easy vector and sequenced. For the amplification of full length clone, gene specific primers were designed from the full length nucleotide sequence as obtained from alignment of partial clone and the 5'RACE product. The full length cDNA clone was amplified by PCR using cDNA as template and the gene specific primer pair (CsULT-F and CsULT-R). The PCR product was run on $1 \%$ agarose gel, purified by gel extraction kit (Qiagen) and subsequently cloned into the pGEM-T Easy vector. The cycling conditions used were $3 \mathrm{~min}$ at $94^{\circ} \mathrm{C}, 30$ cycles (30s at $94^{\circ} \mathrm{C}, 30 \mathrm{~s}$ at $60^{\circ} \mathrm{C}$ and $1 \mathrm{~min}$ at $72^{\circ} \mathrm{C}$ ) and final extension for $10 \mathrm{~min}$ at $72^{\circ} \mathrm{C}$. The nucleotide sequence of CsULT1 was submitted to GenBank and the accession number is KM670459.

\section{Sequence analyses}

The full length nucleotide sequence of CsULT1 was translated using Translate tool (http://web.expasy.org/translate/) and the properties of deduced amino acid sequence were estimated using ProtParam (http://web.expasy. org/protparam/). Multiple sequence alignment and phylogenetic analysis was performed using the ClustalW with the default parameters through the service of the European Bioinformatics Institute (http://www. ebi.ac.uk/Tools/msa/clustalw2).

\section{Subcellular localization}

The subcellular localization of CSULT1 was studied by performing transient expression assay in onion epidermal cells. For this, CsULT1 with restriction sites for NcoI and SpeI was amplified using ULTCam-F and ULTCam$\mathrm{R} 1$ primer pair and fused in frame with 5 ' terminus of GFP reporter gene in pCAMBIA-1302. The cycling parameters were same as described above. The fusion construct of CsULT1-GFP was bombarded on to the onion peels using biolistic gene delivery device PDS-1000/He (Bio-Rad, USA). The onion peels were then incubated for 24 hours before visualizing in confocal microscope.

\section{Transactivation assay}

Full length protein coding sequence of CsULT1 was cloned in yeast (Saccharomyces cerevisiae) expression vector pGBKT7 (Clontech) at NdeI-EcoRI site to express CsULT1 protein fused to GAL4 DNA-binding domain (GAL4-BD). The primers used were ULTGBKT-F and ULTGBKT-R and cycling parameters are same as described above. The resulting construct was transformed into Y187 yeast strain. The positive transformants were selected onto synthetic medium lacking tryptophan and leucine. Cells from two independent transformants were collected and assayed for $\beta$-galactosidase activity by using ortho-nitrophenyl- $\beta$-D-galactoside (ONPG) as substrate as described in clontech manual (PT3024-1).

Plant expression vector and transformation of Crocus calli The CsULT1 gene with NcoI and SpeI restriction sites was PCR amplified using ULTCam-F and ULTCam-R2 primer pair and cloned into pCAMBIA1302 vector, containing 
CaMV 35S promoter. For the transient expression, the Crocus calli were arranged at the center of petri dish and the biolistic gene delivery device PDS-1000/He (Bio-Rad, USA) was used for transgene delivery via microprojectile bombardment. Plasmid DNA (at the concentration of $1 \mu \mathrm{g} / \mu \mathrm{L}$ ) was coated on the surface of gold particles and bombarded on to the calli. The particle delivery system was adjusted to $1100 \mathrm{psi}$ of helium pressure and $27 \mathrm{mmHg}$ of vacuum pressure inside the chamber. After bombardment, the calli were transferred to fresh media and after five days they were again transferred to media containing hygromycin for selection of transgenic structures. After 10 days, the calli which did not harbour the CsULT1-pCAMBIA construct turned blackish whereas the ones with the gene construct looked fresh and were used for further experimental studies. The transgenic calli were screened by genomic PCR. For this, genomic DNA was isolated from independent CsULT1 overexpression and empty control calli using the DNeasy Plant mini kit (Qiagen). The presence of CsULT1 was confirmed by genomic PCR using gene specific primer and reverse primer corresponding to GFP. Transgenic and control calli were used for measuring the transcript levels of few carotenoid pathway genes including PSY (GenBank accession: AJ888514), PDS (GenBank accession: AY183118), BCH (GenBank accession: AJ937791). CCD4b (GenBank accession: EU523663.1) and CCD2 (GenBank accession: KJ541749) using quantitative real time PCR as described above.

\section{Results}

\section{Analysis of Crocin in different tissues and developmental stages}

Since crocin is the most important metabolite in saffron and responsible for its coloring property, we measured its quantity in different parts of Crocus flower and at various stages of stigma development (pre anthesis, anthesis and post anthesis) using HPLC. Results indicated that crocin was present only in stigma part of flower. We were not able to detect crocin in other parts of the flower like tepals and anther. Further, its content showed increasing trend from pre anthesis to anthesis stage and later again decreased after anthesis (Figure 1). This was in confirmation with earlier reports [12] where they have shown that the major apocarotenoids like crocin and picrocrocin are detected in orange stage and increased rapidly during the following stages of stigma development till they reached maximum in scarlet stage at anthesis. This confirms that stigma is the site for synthesis and accumulation of major Crocus apocarotenoids and their synthesis is congruent with development of stigma reaching highest at anthesis stage.

\section{Isolation and expression profiling of CsULT1}

We aimed at identification of transcription factors which regulate biosynthesis of Crocus apocarotenoids.
Towards this, five transcription factors belonging to different gene families were selected from saffron ESTs (www.ncbi.nlm.nih.gov/nucest). The selected genes were Myb (cr.saCl000348:1), MADS box (cr.saCl001329:1), WRKY (cr.saCl000652:1), Zinc finger (cr.saCl000359) and ULT (cr.saCl000502:1). The expression pattern for all these genes was investigated in various tissue types and at different developmental stages using quantitative real time PCR. Our results demonstrated that a $U L T$ transcription factor showed higher induction in stigma part of the flower and its expression increased till the day of anthesis and then subsequently decreased (Figure 2a and b). This expression pattern corroborated with the accumulation pattern of apocarotenoids suggesting involvement of this gene in regulating biosynthesis of these compounds. Among other genes studied, only $M y b$ showed higher expression in stigma as compared to other flower parts, however, its expression at different developmental stages of stigma did not match with the pattern of apocarotenoid accumulation (Additional file 3).

Full length $U L T$ was cloned by RT- PCR and 5' RACE and was named as CsULT1 [GenBank accession number: KM670459]. The gene contains 708 bp open reading frame coding for 235 amino acids long protein (Additional file 4) with a predicted molecular mass of $26.5 \mathrm{kD}$ and $\mathrm{pI} 8.32$. Domain search revealed presence of conserved SAND domain in CSULT1 which normally consists of evolutionarily conserved 80 to 100 amino acid long DNA binding motif [18]. The sequence alignment of ULTs from various organisms has revealed two conserved cores in SAND domains viz TPxxFE and KDWK. While TPxxFE is perfectly conserved among all ULT proteins in plants, KDWK shows variability at primary level however, the secondary structure is conserved [19]. Alignment of CsULT1 with other plant ortholgs (Figure 3) showed high sequence homology with ULT from Phoenix (79.57\%), Vitis vinifera (78\%), Populus (76\%) and Medicago (75\%). Sequence alignment showed that ULT proteins show significant homology along the entire length of the protein except at the extreme $\mathrm{N}$ terminus. Moreover, TPxxFE motif was present in CsULT1 and was conserved among all the proteins used for alignment. Phylogenetic analysis of selected sequences placed CsULT1 close to its homolog from Phoenix (Figure 4).

\section{Subcellular localization of CsULT1}

In order to have a preliminary understanding about the mechanism underlying the regulatory activity of CsULT1, its subcellular localization was investigated. Programs like Prosite and PSORT revealed absence of any sorting signal and predicted CsULT1 to be localized in cytosol. For confirming the localization experimentally, CsULT1 was cloned in frame with GFP reporter gene. The expression of the fusion gene construct CsULT1-GFP was driven by the $35 \mathrm{~S}$ promoter of cauliflower mosaic virus ( $\mathrm{CaMV}$ - 
A mau

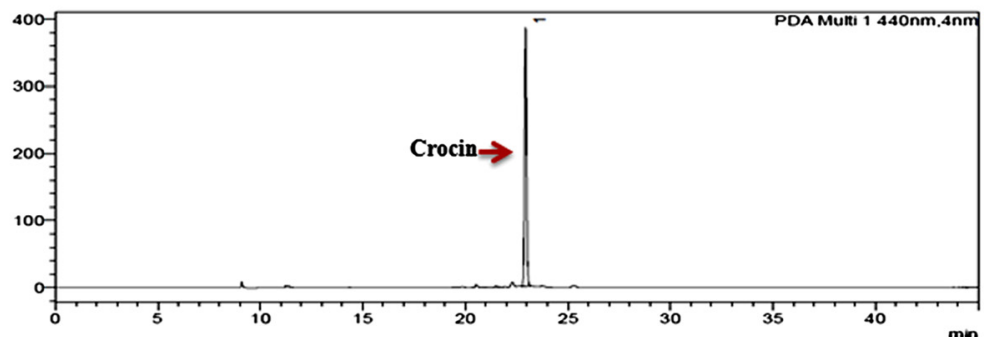

B

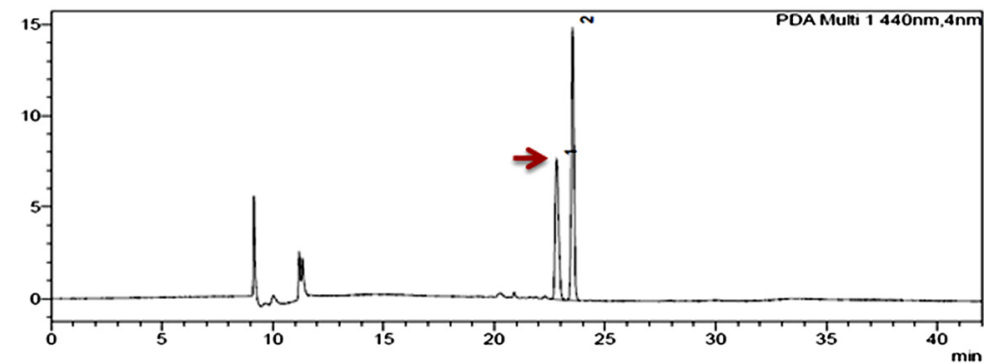

C

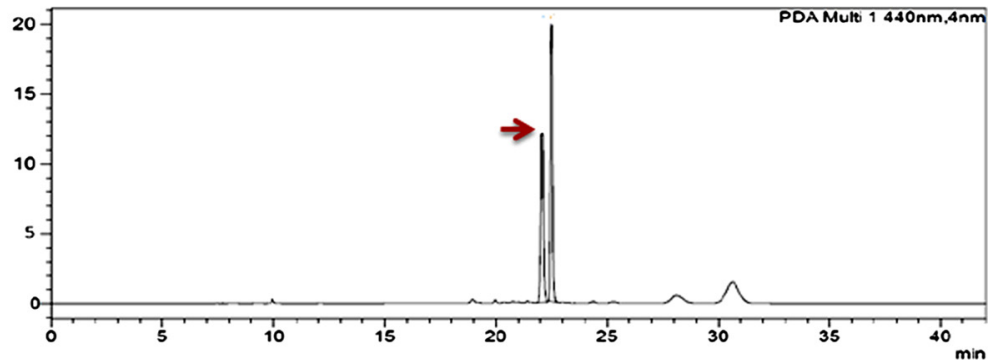

D

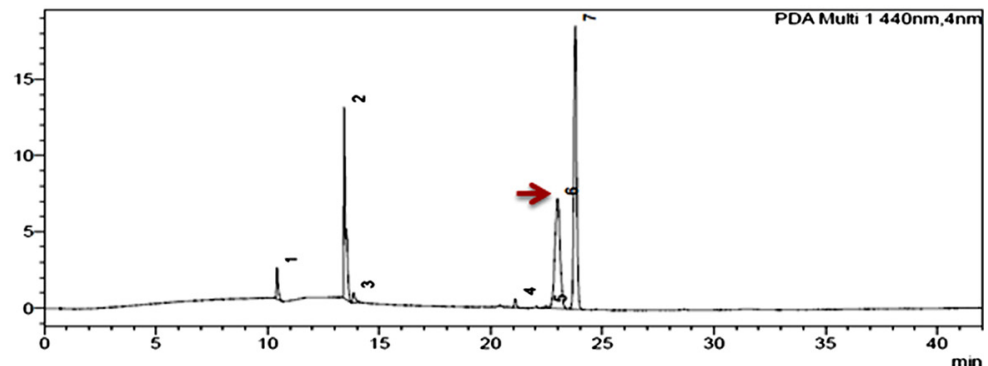

Figure 1 HPLC chromatograms for crocin. (A) represents chromatogram for crocin standard, (B-D) chromatogramas for Crocus stigma collected at pre anthesis, anthesis and post anthesis stages.

$35 S$ ). The fusion gene was introduced into onion (Allium cepa) epidermal cells by particle bombardment. While the control GFP accumulated throughout the cell, CsULT1GFP was localized in the nucleus (Figure 5). This might be because of the fact that ULT proteins are small enough and can diffuse passively into the nucleus through the nuclear pores [15].

\section{Transactivation assay}

To investigate the ability of CsULT1 to activate transcription, a transient expression assay was performed using a GAL4-responsive reporter system in yeast cells. For this, the full-length coding region of CsULT1 was fused to the GAL4 DNA-binding domain (BD) to generate pGBKT7-CsULT1-BD construct which was then transformed into yeast strain Y187. The transformants were assayed for their ability to activate transcription from the GAL4 upstream activation sequence. The transformed yeast cells harboring pGBKT7-CsULT1-BD construct grew well in SD medium lacking tryptophan and leucine, and showed $\beta$-galactosidase activity, whereas cells containing pGBKT7 (negative control) showed no $\beta$-galactosidase activity (Figure 6). This data confirmed transcriptional activity of CSULT1.

\section{Induction of CsULT1 by phytohormones}

To investigate the effect of various phytohormones on expression of CsULT1, Crocus flowers were treated with 

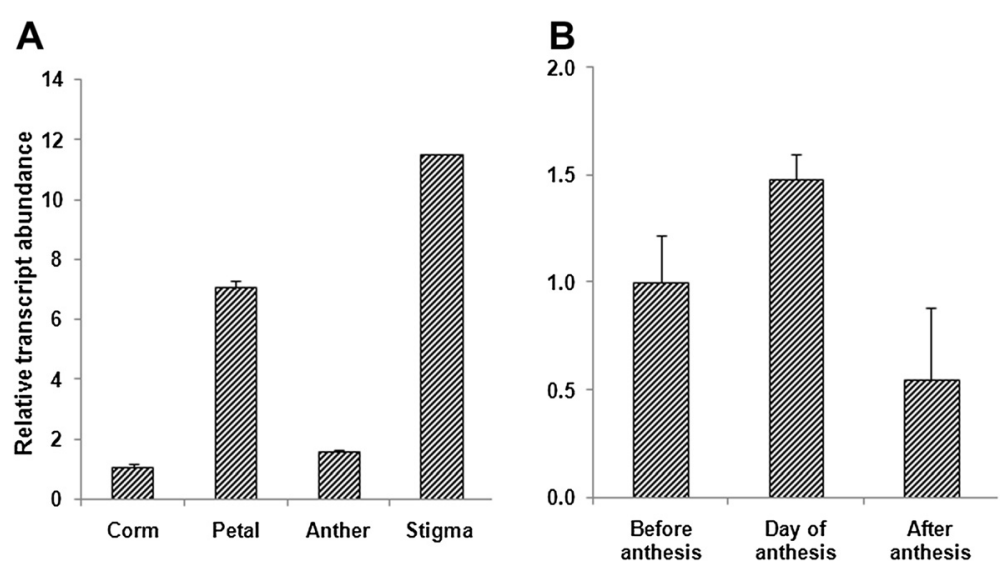

Figure 2 qRT-PCR analysis of CsULT1 expression. (A) in different tissues of C. sativus (B) at different developmental stages. Transcript levels were normalized by actin. Data are means and SD from three biological replicates.

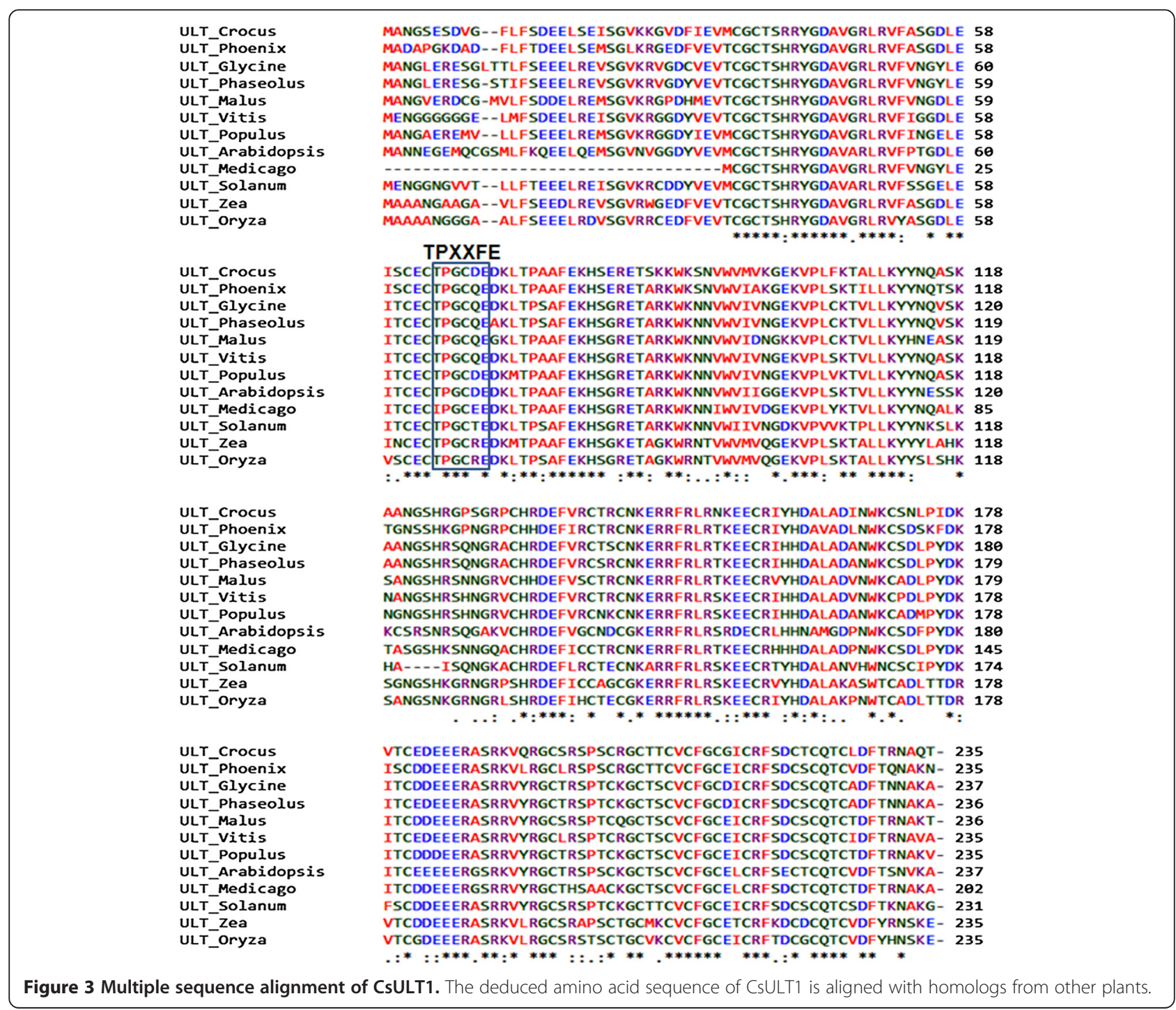




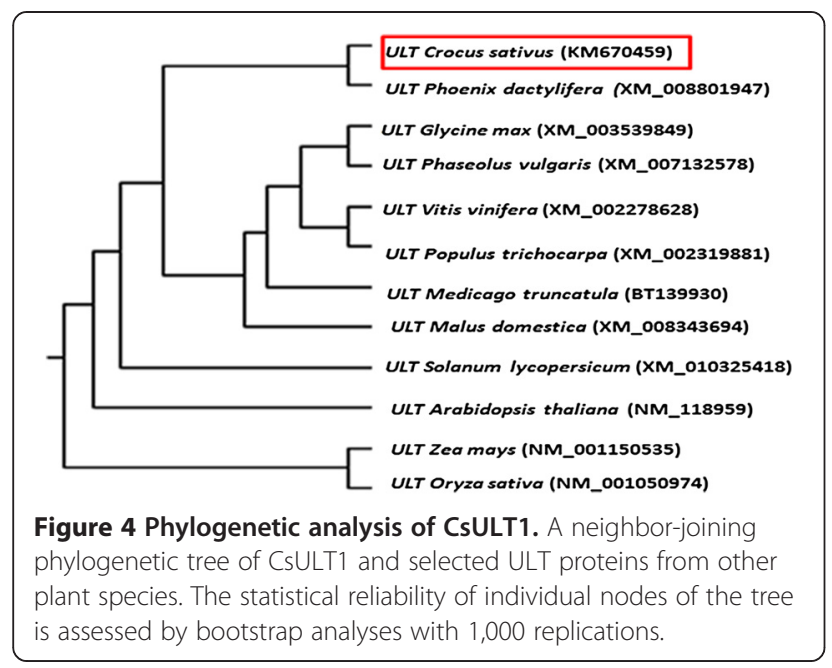

salicylic acid (SA), methyljasmonate (JA), 2,4-D and abscisic acid (ABA) in a time course study. The expression of CsULT1 was measured by qPCR using RNA isolated from treated tissue samples. Compared with the uninduced control, CsULT1 expression increased in response to all these hormones used (Figure 7). In response to SA treatment, expression of CsULT1 increased upto 122 fold ( $\log 7$ fold) at 24 hr post treatment while in response to JA, the expression enhanced approximately upto 150 fold (log 7 fold) at $12 \mathrm{~h}$ post treatment. CsULT1 showed maximum change in expression in response to 2,4-D where it showed 175 fold (log 7.4) induction at $12 \mathrm{hr}$ of treatment. However, there was not much significant change in expression in response to $\mathrm{ABA}$.

\section{Transient over-expression of CsULT1 in C. sativus calli increases MEP pathway gene expression}

For gaining an understanding on the role of CsULT1 in Crocus apocarotenoid biosynthesis, the gene was transiently expressed in Crocus calli under the control of CaMV-35S promoter. The presence of transgene in transiently transformed calli was confirmed by genomic PCR. In the CsULT1-overexpressing calli, the CsULT1 gene was expressed 2.5 fold higher than the empty vector control. Further, we checked expression of few of the MEP pathway genes and observed that PSY, PDS, $B C H$, $C s C C D 4 b$ and $C s C C D 2$ genes showed upregulation in CsULT1 overexpressing calli (Figure 8a). PSY and $P D S$ catalyze the initial rate limiting steps in carotenoid biosynthetic pathway. Further, $B C H$ is involved in the formation of zeaxanthin from beta carotene [11] and this zeaxanthin acts as the substrate for the formation of Crocus apocarotenoids by CsCCD2 enzyme [20]. $C s C C D 4 b$ (another member of CCD gene family) is also involved in the formation of apocarotenoids from carotenoid substrates. Therefore, enhanced expression of the

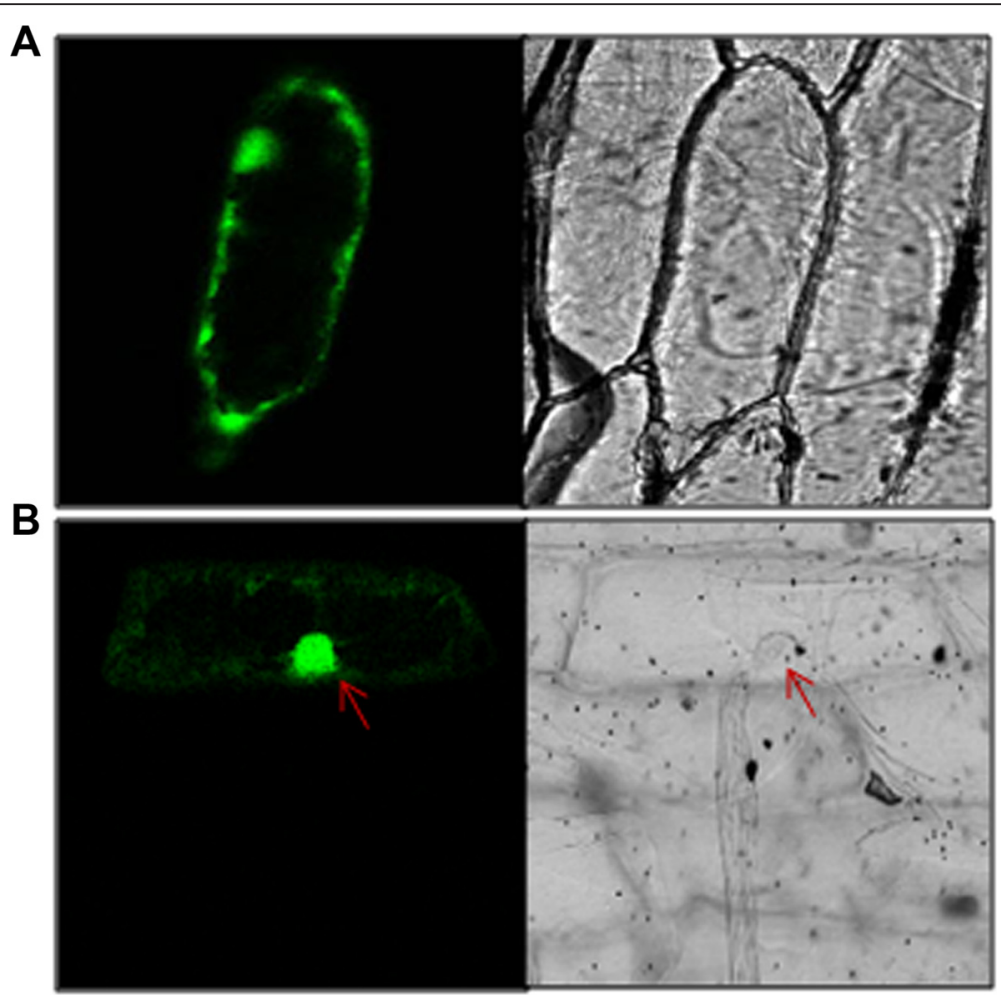

Figure 5 Subcellular localization of CsULT1. (A) GFP is accumulated throughout the cell (B) CSULT1-GFP is localized to the nucleus. 


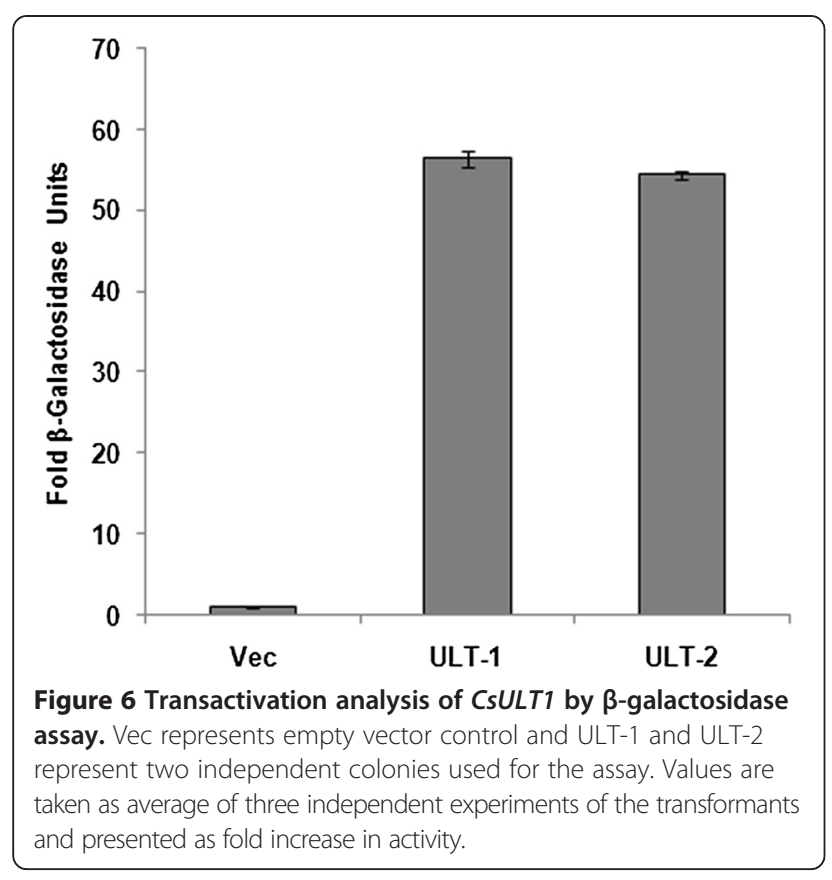

above mentioned genes may result in increased zeaxanthin pool which may subsequently be tailored to form apocarotenoids.

Various phytohormones were shown to induce expression of CsULT1 which in turn induced expression of key pathway genes of carotenoid metabolism. Therefore we were keen to investigate change in expression of pathway genes in response to phytohormone application. It was observed that SA, JA and 2,4-D induced expression of carotenoid pathway genes (Figure $8 \mathrm{~b}$ ) therefore indicating their possible role in mediating the function of CsULT1 in regulating carotenoid/apocarotenoid biosynthesis. Taken together, these results suggest that CsULT1 has a role in regulating metabolic flux towards the biosynthesis of apocarotenoids in Crocus.

\section{Discussion}

Crocus is known to accumulate large amounts of the apocarotenoids like crocetin (and its glycosylated forms, crocins), picrocrocins and saffranal in stigma part of the flowers. The proposed biosynthetic pathway is initiated through the symmetric cleavage of zeaxanthin at the 7,8 / 7,8 positions by a CCD2 enzyme [20]. The two cleavage products formed are $3-\mathrm{OH}-\beta$-cyclocitral and crocetin

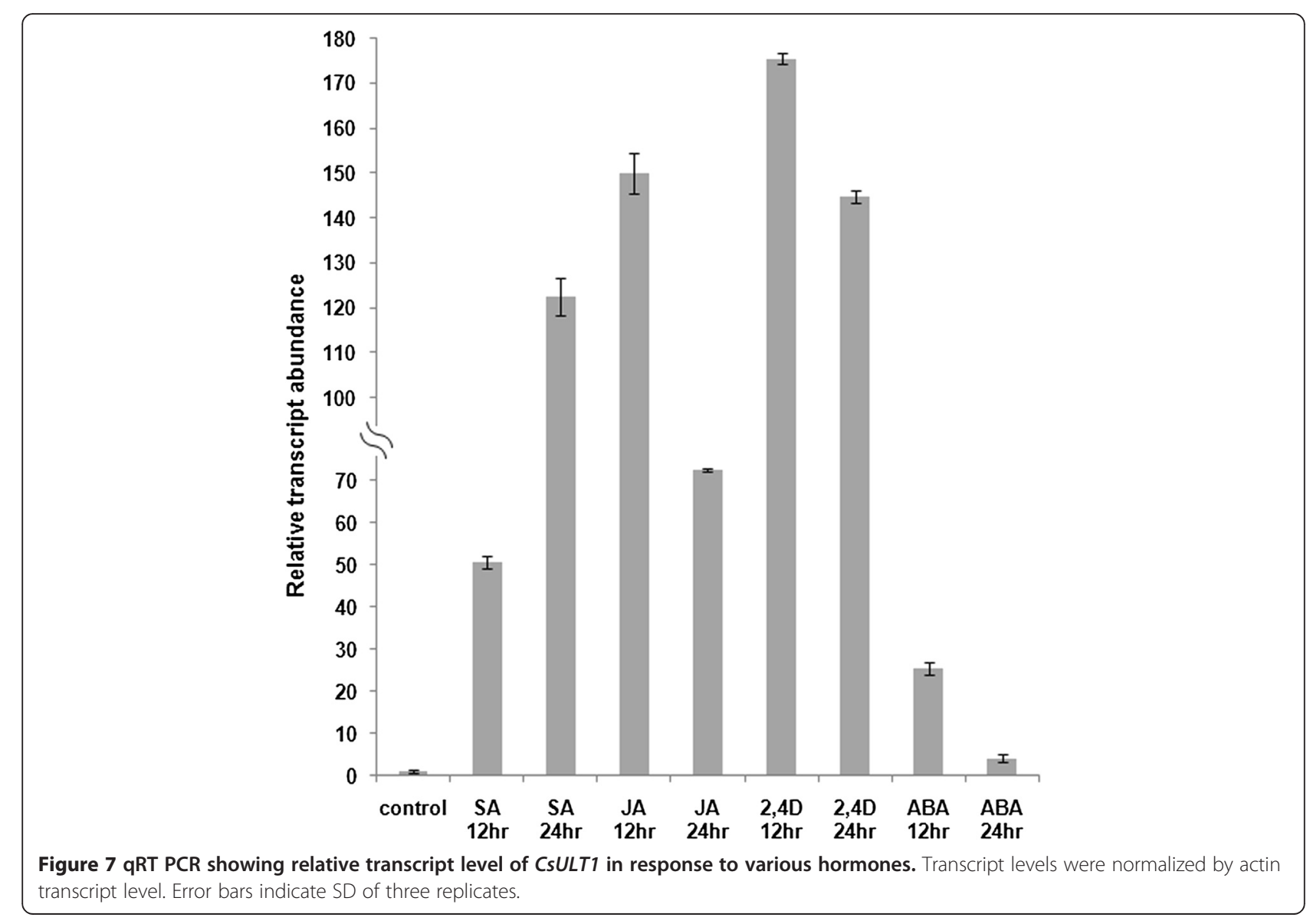




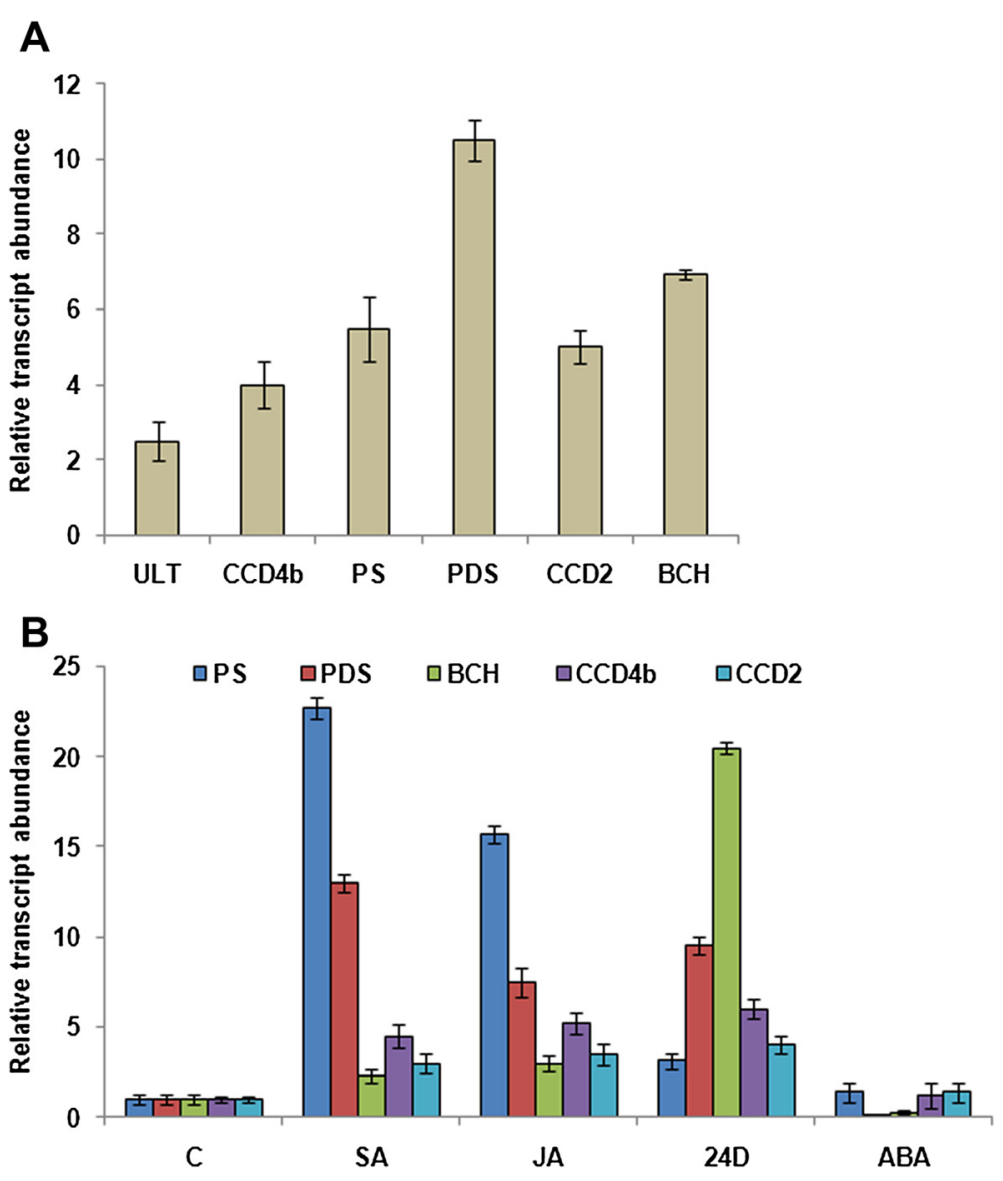

Figure 8 Relative expression levels of selected carotenoid pathway genes (A) in CsULT1 overexpressing calli (B) in response to various phytohormones. The actin gene was used as an internal control. Each relative gene expression represents the average of three replicates with error bars representing SD.

dialdehyde which are further dehydrogenated and glycosylated to yield picrocrocin and crocins respectively. Aim of our study was to identify transcription factors regulating synthesis of these Crocus apocarotenoids. For this, we used combined approach of transcript and metabolite profiling. Since crocetin (which is subsequently converted into crocin) and picrocrocin are products of same cleavage step and crocin is more stable, we investigated crocin levels in different parts of Crocus flower and in stigma collected at three different developmental stages (pre anthesis, anthesis and post anthesis). We could detect crocin only in stigma while in other parts it was below detection levels. Further, crocin content increased from pre anthesis stage to anthesis and later decreased post anthesis (Figure 1). In earlier reports also same trend has been described for crocin accumulation [12]. Next, expression profile of CsULT1 was investigated which indicated that it follows the same trend (Figure 2) and corroborates with accumulation pattern of crocin suggesting its possible role in regulating the crocin biosynthetic pathway. The apocarotenoid accumulation varies with developmental stages and thus in order to fit into this narrow window of developmental changes, chromatin needs to be flexibly regulated so as to confer stable expression states that can be reset owing to changes in the progression of development. Since recently ULT has been shown to act in chromatin mediated pathways, its involvement in regulating plant secondary metabolic pathways would be a new dimension to its functional domain.

Domain analysis showed that CsULT1 contains a SAND domain which represents conserved 80-residue amino acid sequence and is found in a number of nuclear proteins, many of which function in chromatin-dependent transcriptional control [13]. These include proteins linked to various human diseases, such as the Sp100 (Speckled protein $100 \mathrm{kDa}$ ), NUDR (Nuclear DEAF-1 related), GMEB (Glucocorticoid Modulatory Element Binding) proteins and AIRE-1 (Autoimmune regulator 1) proteins [18]. Many of these proteins have been shown to bind DNA, but no clear sequence or structural relationship to known DNA binding motifs has been established. Based on the conservation of positively charged residues, including a characteristic 
KDWK sequence motif, the SAND domain has been suggested to mediate the DNA binding of these proteins.

In animals SAND domain-containing proteins are found in nucleus or cytoplasm, or have dual localization being present in both nucleus and cytoplasm [21-23]. In confirmation with this data pertaining to animal proteins, plant ultrapetala proteins with SAND domains are also demonstrated to localize to both the nucleus and the cytosol [15]. However, our study showed that CsULT1 is localized in nucleus (Figure 5). SAND domain containing proteins have been found to be transcriptionally active and are involved in regulation of gene expression [17]. We also investigated transcriptional activity of CsULT1 and our results demonstrated that it activated beta galactosidase enzyme proving that it is transcriptionally active (Figure 6).

Plant developmental, metabolic and stress pathways have been shown to be influenced and controlled by various phytohormones. In order to gain an insight about effect of different phytohormones on CsULT1 expression, we investigated effect of SA, JA, 2,4D and ABA on expression of CsULT1. Our results indicated that CsULT1 is induced in response to all the hormones studied. However, effect of SA, JA and 2,4-D was much more profound than ABA (Figure 7). Jasmonates are known elicitors of plant secondary metabolism and trigger extensive transcriptional reprogramming which ultimately leads to activation of whole metabolic pathway [24]. Induction of CsULT1 in response to JA might be part of this transcriptional activation scenario which as a final outcome leads to activation of carotenoid metabolic pathway. SA has been reported to induce expression of many carotenogenesis related genes [25]. Since CsULT1 is a probable regulator of carotenogenesis, its induction in respone to SA is thus in confirmation with earlier reports. Auxin has been demonstrated to have a profound effect on stigma development [26]. Therefore enhanced expression of CsULT1 in response to 2,4-D treatment might suggest a parallel role of auxin in regulating stigma development vis a vis apocarotenoid biosynthesis in Crocus. Although ABA treatment also enhanced expression of CsULT1, it was much less as compared to other hormones.

Several attempts have been made to establish Crocus transformation but no success has been achieved so far. Lack of transformation protocol is a limitation for functional characterization of genes in Crocus. The site of apocarotenoid biosynthesis is Crocus stigma, however, many of the pathway genes are expressed in callus also. Considering the limitation of transformation system, we transiently overexpressed CsULT1 in Crocus calli by particle bombardment in order to confirm its role in regulating carotenoid/apocarotenoid pathway. The expression of CsULT1 in transgenic calli was 2.5 fold as compared to vector control. This value is not good enough but since transformation in Crocus has not been established and callus is not the actual site of gene expression, the reported increase in expression can be considered as significant. Further, the expression analysis of a few carotenoid pathway genes was carried out and the results showed increase in expression level of PSY and PDS genes which catalyze initial rate limit steps of this pathway. This suggests role of CsULT1 in regulating carotenoid biosynthesis in Crocus. Till so far there is only one report on regulation of $P S Y$ gene expression by phytochrome interacting factor (PIF1) which binds directly to PSY promoter and thereby regulates carotenoid accumulation during daily cycles of light and dark in mature plants [27]. Another member of AP2 gene family (RAP2.2) binds to of $P S Y$ promoter and is shown to modestly regulate the transcript levels of $P S Y$ and $P D S$ in Arabidopsis [28]. Also $B C H$ which is involved in conversion of beta carotene into zeaxanthin showed enhanced expression in transgenic calli (Figure 8a). In earlier reports $C s C C D 4 b$ was considered responsible for cleaving zeaxanthin to produce apocarotenoids. However, later it was shown to cleave beta carotene at the 9,10 and/ or the 9,10 positions, yielding beta-ionone. Recently a new isoform of CCDs (CsCCD2) was identified and isolated from Crocus and was shown to cleave zeaxanthin sequentially at 7,8 and 7,8 double bonds suggesting that $C s C C D 2$ catalyzes the first dedicated step in crocin biosynthesis [20]. We investigated change in expression of $C s C C D 4 b$ as well as $C s C C D 2$ in transgenic calli and observed that their expression was enhanced around 4 and 5 fold respectively (Figure 8a). This suggests that apart from regulating synthesis of crocin and picrocrocin from zeaxanthin, CsULT1 also plays role in regulating biosynthesis of other apocarotenoids including beta ionone. Thus CsULT1 might regulate expression of more than one members of $C C D$ gene family. Except for PIF1 and RAP2.2 no other transcription factors have been identified till so far which regulate expression of genes involved in carotenogenesis in plants. Therefore the present work will form a platform for enhancing our knowledege on regulation of this important pathway.

Carotenoids are involved in many biological functions including plastid biogenesis, photosynthesis, photomorphogenesis etc. Carotenoid metabolic pathway is also linked with many other pathways like ABA and GA biosynthesis. Therefore, carotenoid metabolism might be regulated at multifaceted levels in plants. Further, because of this close coordination of many pathways, content and composition of carotenoids is important. Thus biosynthesis of carotenoids and their turn-over to produce apocarotenoids needs to be tightly regulated in order to maintain their steady levels in plants. Hormones are known to play key roles in regulating various metabolic pathways. They also help in coordinating 
interplay between various pathways. Our results also demonstrated effect of phytohormones on CsULT1 expression. In order to further our understanding on mechanism of regulation of carotenoid biosynthesis by CsULT1, we also investigated effect of various phytohormones on carotenoid pathway genes particularly those which showed enhanced expression in CsULT1 overexpressing calli. Our results indicated that expression of all the genes studied was enhanced in response to SA, JA and 2, 4-D, however, there was no significant increase in expression of these genes in response to $\mathrm{ABA}$ (Figure $8 \mathrm{~b}$ ). Earlier also, SA and JA have been shown to affect expression of many carotenogenic pathway genes [29]. If considered individually, $P S Y$ was induced more in response to SA and JA while PDS expression increased more under the influence of SA and 2,4-D. $B C H$ showed much higher response to 2,4-D than other hormones while $C C D 4 b$ and $C C D 2$ showed more or less similar trend in expression in response to all the hormones except ABA. ABA did not cause any significant effect on expression of carotenoid pathway genes. Earlier, exogenous application of ABA was shown to repress transcript levels of many chloroplast genes [30]. It was also found to reduce chlorophyll levels and also endogenous ABA levels [31]. This might be the reason that we did not observe any significant increase in expression levels of carotenoid pathway genes in response to ABA. Thus in general, change in the expression of these genes followed the same trend as that of CsULT1. This suggests that these phytohormones might play a role in mediating effect of CsULT1 on regulation of carotenoid metabolism. Further, different genes showed varied induction levels in response to different hormones which might be because of the fact that control of plant metabolic pathways is a complex phenomenon and network of cross-communicating hormone signaling pathways are involved so as to maintain overall metabolite homeostasis within the plant system.

Taken together, these results suggest that CsULT1 plays role in diverting metabolic flux towards enhanced production of apocarotenoids. Recently ULT in Arabidopsis has been shown to be involved in gynoecium development and patterning [17]. In Crocus, apocarotenoid biosynthesis is congruent with stigma development. This indicates that CsULT1 might have a parallel role in regulating Crocus gynoecium (stigma) development vis a vis apocarotenoid biosynthesis. However, this needs to be further verified by experimentation in Crocus itself or in other alternative plant system.

\section{Conclusions}

In this study an ultrapetala transcription factor from Crocus, CsULT1, was identified, cloned and characterized. The CsULT1 transcript was expressed more in stigma till flower anthesis. Application of phytohormones like 2,4D, JA and SA enhanced CsULT1 expression. The gene is nuclear localized and is transcriptionally active. Moreover, ectopic expression of this gene altered expression of some important genes of carotenoid/apocarotenoid pathway confirming that CsULT1 plays a regulatory role in Crocus apocarotenoid biosynthesis. Furthermore, the hormones which affected expression of CsULT1 were also shown to enhance expression of pathway genes indicating their role in mediating regulatory role of CsULT1.

\section{Additional files}

Additional file 1: Table S1. List of primer sequences.

Additional file 2: Figure S1. Melt curve depicting single peak in qRT-PCR.

Additional file 3: Figure S2. qRT-PCR analysis of different Crocus transcription factors.

Additional file 4: Figure S3. Nucleotide and deduced amino acid sequence of CSULT1.

\section{Abbreviations}

ULT: Ultrapetala; PSY: Phytoene synthase; PDS: Phytoene desaturase; CCD: Carotenoid cleavage dioxygenase; $\mathrm{BCH}$ : Beta cyclohydroxylase; MEP: Methylerythritol phosphate.

\section{Competing interests}

The authors declare that they have no competing interests.

\section{Authors' contributions}

NA: designed and performed experiments and wrote the manuscript; DJ: performed experiments; RAV: conceptualized experiments and did critical revision of manuscript. All authors read and approved the final manuscript.

\section{Acknowledgements}

This work was supported by grants SIMPLE (BSC-0109) from CSIR and GAP-1177 from DST, Government of India.

\section{Author details}

${ }^{1}$ Plant Biotechnology Division, CSIR- Indian Institute of Integrative Medicine, Sanat Nagar, Srinagar J\&K-190005, India. ${ }^{2}$ National Institute of Plant Genome Research, Aruna Asaf Ali Marg, New Delhi 110067, India. ${ }^{3}$ Medicinal

Chemisrty Division, CSIR- Indian Institute of Integrative Medicine, Canal Road, Jammu J\&K 180001, India.

Received: 1 October 2014 Accepted: 14 January 2015

Published online: 01 February 2015

\section{References}

1. D'Agostino N, Pizzichini D, Chiusano ML, Giuliano G. An EST database from saffron stigmas. BMC Plant Biol. 2007;7:53.

2. Auldridge ME, McCarty DR, Klee HJ. Plant carotenoid cleavage oxygenases and their apocarotenoid products. Curr Opin Plant Biol. 2006;9:315-21.

3. Liao YH, Houghton PJ, Hoult JR. Novel and known constituents from Buddleja species and their activity against leukocyte eicosanoid generation. J Nat Prod. 1999:62:1241-5.

4. Pfander $\mathrm{H}$, Schurtenberger $\mathrm{H}$. Biosynthesis of C20-carotenoids in Crocus sativus. Phytochemistry. 1982;21:1039-42.

5. Rubio A, Rambla JI, Santaella M, Gomez MD, Orzaez D, Granell A, et al. Cytosolic and plastaglobule targeted Carotenoid dioxygenases from Crocus sativus are both involved in B-ionone-release. J Biol Chem. 2008;283:24816-25.

6. Winterhalter P, Rouseff RL. Carotenoid-derived aroma compounds. Washington, DC: American Chemical Society; 2002.

7. Abdullaev Fl, Espinosa-Aguirre JJ. Biomedical properties of saffron and its potential use in cancer therapy and chemoprevention trials. Cancer Detect Prev. 2004;28:426-32. 
8. Zhang Z, Wang CZ, Wen XD, Shoyama Y, Yuan CS. Role of saffron and its constituents on cancer chemoprevention. Pharm Biol. 2013;51:920-4.

9. Cunningham FX, Gantt E. Genes and enzymes of carotenoid biosynthesis in plants. Annu Rev Plant Physiol Plant Mol Biol. 1998:49:557-83.

10. Lu S, Li L. Carotenoid metabolism: biosynthesis, regulation and beyond. J Integr Plant Biol. 2008;50:778-85.

11. Castillo R, Fernandez J, Gomez-Gomez L. Implications of carotenoid biosynthetic genes in apocarotenoid formation during the stigma development of crocus sativus and its closer relatives. Plant Physiol. 2005;139:674-89.

12. Moraga AR, Rambla JL, Ahrazem O, Granell A, Gómez-Gómez L. Metabolite and target transcript analyses during Crocus sativus stigma development. Phytochemistry. 2009;70:1009-16.

13. Bottomley MJ, Collard MW, Huggenvik JI, Liu Z, Gibson TJ, Sattler M. The SAND domain structure defines a novel DNA-binding fold in transcriptional regulation. Nat Struct Biol. 2001;8:626-33.

14. Fletcher JC. The ULTRAPETALA gene controls shoot and floral meristem size in Arabidopsis. Development. 2001;128:1323-33.

15. Carles CC, Lertpiriyapong K, Reville K, Fletcher JC. The ULTRAPETALA1 gene functions early in Arabidopsis development to restrict shoot apical meristem activity, and acts through WUSCHEL to regulate floral meristem determinacy. Genetics. 2004;167:1893-903.

16. Carles CC, Fletcher JC. The SAND domain protein ULTRAPETALA1 acts as a trithorax group factor to regulate cell fate in plants. Genes Dev. 2009:23:2723-8

17. Monfared MM, Carles CC, Rossignol P, Pires HR, Fletcher JC. The ULT1 and ULT2 trxG genes play overlapping roles in Arabidopsis development and gene regulation. Mol Plant. 2013;6:1564-79.

18. Gibson TJ, Ramu C, Gemünd C, Aasland R. The APECED polyglandular autoimmune syndrome protein, AIRE-1, contains the SAND domain and is probably a transcription factor. Trends Biochem Sci. 1998;23:242-4.

19. Carles CC, Fletcher JC. Missing links between histones and RNA Pol II arising from SAND? Epigenetics. 2010;2010(5):381-5.

20. Frusciante S, Diretto G, Bruno M, Ferrante P, Pietrella M, Prado-Cabrero A et al. Novel carotenoid cleavage dioxygenase catalyzes the first dedicated step in saffron crocin biosynthesis. PNAS. 2014;111:12246-51.

21. Gross CT, McGinnis W. DEAF-1, a novel protein that binds an essential region in Deformed response element. EMBO J. 1996;15:1961-70.

22. Jimenez-Lara AM, Heine MJ, Gronemeyer $\mathrm{H}$. Cloning of a mouse glucocorticoid modulatory element binding protein, a new member of the KDWK family. FEBS Lett. 2000;468:203-10.

23. Peterson P, Pitkänen J, Sillanpaa N, Krohn K. Autoimmune polyendocrinopathy candidiasis ectodermal dystrophy (APECED): a model disease to study molecular aspects of endocrine autoimmunity. Clin Exp Immunol. 2004;135:348-57.

24. De Geyter N, Gholami A, Goormachtig S, Goossens A. Transcriptional machineries in jasmonate-elicited plant secondary metabolism. Trends Plant Sci. 2012;176:349-59.

25. Gao Z, Meng C, Zhang X, Xu D, Miao X, Wang Y, et al. Induction of salicylic acid (SA) on transcriptional expression of eight carotenoid genes and astaxanthin accumulation in Haematococcus pluvialis. Enzyme Microb Technol. 2012;51:225-30.

26. Sundberg E, Østergaard L. Distinct and dynamic auxin activities during reproductive development. 2012. Published by Cold Spring Harbor Laboratory Press

27. Toledo-Ortiz G, Huq E, Rodríguez-Concepción M. Direct regulation of phytoene synthase gene expression and carotenoid biosynthesis by phytochrome-interacting factors. PNAS. 2010;107:11626-31.

28. Welsch R, Maass D, Voegel T, DellaPenna D, Beyer P. Transcription factor RAP2.2 and its interacting partner SINAT2: stable elements in the carotenogenesis of Arabidopsis leaves. Plant Physiol. 2007;145:1073-85.
29. Gao Z, Meng C, Zhang X, Xu D, Zhao Y, Wang Y, et al. Differential expression of carotenogenic genes, associated changes on astaxanthin production and photosynthesis features induced by JA in $\mathrm{H}$. pluvialis. PloS One. 2012;7:e42243.

30. Yamburenko MV, Zubo YO, Vanková R, Kusnetsov W, Kulaeva ON, Börner T. Abscisic acid represses the transcription of chloroplast genes. J Exp Bot. 2013;64:4491-502

31. Meier S, Tzfadia O, Vallabhaneni R, Gehring C, Wurtzel ET. A transcriptional analysis of carotenoid, chlorophyll and plastidial isoprenoid biosynthesis genes during development and osmotic stress responses in Arabidopsis thaliana. BMC Syst Biol. 2011;5:77.

\section{Submit your next manuscript to BioMed Central and take full advantage of:}

- Convenient online submission

- Thorough peer review

- No space constraints or color figure charges

- Immediate publication on acceptance

- Inclusion in PubMed, CAS, Scopus and Google Scholar

- Research which is freely available for redistribution 